\title{
Diretrizes curriculares nacionais dos cursos de graduação em Terapia Ocupacional: especificidade e competências profissionais
}

\author{
National curriculum guidelines for degree programmes in Occupational Therapy: \\ specificity and professional competences
}

\author{
Marília Meyer Bregalda $^{1}$ e Elisabete Ferreira Mângia ${ }^{2}$
}

http://dx.doi.org/10.11606/issn.2238-6149.v31i1-3p78-85

\begin{abstract}
Bregalda MM, Mângia EF. Diretrizes curriculares nacionais dos cursos de graduação em Terapia Ocupacional: especificidade e competências profissionais. Rev Ter Ocup Univ São Paulo. 2020 jan.-dez.;31(1-3):78-85.
\end{abstract}

RESUMO: Introdução: As Diretrizes Curriculares Nacionais do Curso de Graduação em Terapia Ocupacional definem os princípios para organização e desenvolvimento dos projetos pedagógicos e propõem competências específicas para terapeutas ocupacionais. Objetivos: Identificar e compreender a especificidade e identidade profissionais presentes nas 34 competências apresentadas como específicas para terapeutas ocupacionais pelo documento. Método: A partir da análise das diretrizes, essas competências foram sistematizadas de acordo com sua especificidade profissional, e elaboraram-se sínteses descritivas com suas características e fragilidades. Resultados: Das 34 competências, apenas quatro singularizam a profissão, diferenciando-a das demais. Discussão: A fragilidade na definição das competências específicas faz com que elas estabeleçam poucas relações com os objetos e objetivos da profissão e não permitam identificar, credenciar ou avaliar o perfil profissional. Conclusão: O principal desafio da reformulação das diretrizes nacionais é definir competências que contemplem a mudança paradigmática e a identidade profissional construída a partir dela, de forma a conferir especificidade às competências desenvolvidas na formação em Terapia Ocupacional.

DESCRITORES: Terapia ocupacional; Competência profissional; Formação profissional; Currículo; Diretrizes.
Bregalda MM, Mângia EF. National curriculum guidelines for degree programmes in Occupational Therapy: specificity and professional competences. Rev Ter Ocup Univ São Paulo. 2020 Jan-Dec;31(1-3):78-85.

ABSTRACT: Introduction: The National Curricular Guidelines for the Undergraduate Course in Occupational Therapy define the principles for the organization and development of pedagogical projects and propose specific competences for occupational therapists. Objectives: To identify and understand the professional specificity and identity present in the 34 competences presented as specific to occupational therapists by the document. Method: Based on the analysis of the guidelines, these competences were systematized according to their professional specificity, and descriptive syntheses were elaborated, with their characteristics and weaknesses. Results: Of the 34 competences, only four distinguish the profession, differentiating it from the others. Discussion: The weakness in the definition of specific competences means that they establish few relations with the objects and objectives of the profession and do not allow the identification, accreditation or evaluation of the professional profile. Conclusion: The main challenge of the reformulation of national guidelines is to define competences that contemplate the paradigmatic change and the professional identity built from it, in order to give specificity to the competences developed in the training in Occupational Therapy.

KEYWORDS: Occupational therapy; Professional competence; Professional training; Curriculum; Guidelines.

O artigo é parte da pesquisa de doutorado intitulada "Formação graduada em Terapia Ocupacional e desenvolvimento de competências relacionais: estudo dos currículos dos cursos públicos no Brasil", desenvolvida pela autora principal. A pesquisa não contou com nenhuma fonte de financiamento institucional.

1. Professora Adjunta do Curso de Terapia Ocupacional da Universidade Federal de Paraíba. Departamento de Terapia Ocupacional - Centro de Ciências da Saúde - Universidade Federal da Paraíba. Campus I - Cidade Universitária. E-mail: marilia.bregalda@academico.ufpb.br

2. Livre docente pela Faculdade de Medicina da Universidade de São Paulo e Professora Associada do Curso de Terapia Ocupacional da Faculdade de Medicina da Universidade de São Paulo, São Paulo/SP E-mail: mangeli@usp.br. Orcid: 0000-0002-2572-6639.

Endereço para correspondência: Marília Meyer Bregalda. Bregalda MM. Departamento de Terapia Ocupacional - Centro de Ciências da Saúde Universidade Federal da Paraíba. Campus I - Cidade Universitária. CEP 58051-900. João Pessoa/PB, Brasil. E-mail: marilia.bregalda@academico.ufpb.br. 


\section{INTRODUÇÃO}

$\mathrm{D}$ esdeos anos 1990e, mais fortemente, nos anos 2000, o desafio da formação de profissionais para o Sistema Único de Saúde (SUS) se configura como questão central na formulação das políticas de formação de recursos humanos em saúde. Este desafio esteve presente no processo de construção do SUS, resultou no compromisso com as mudanças nas graduações e afirmou a importância da integração ensino-serviço-comunidade. A Constituição Federal de 1988, no artigo 200, e a Lei Orgânica da Saúde, nos artigos 15 e 27, determinam que são atribuições do SUS a ordenação da formação dos profissionais da saúde e a realização de pesquisas e estudos na área ${ }^{1-5}$.

Para tanto, fez-se necessária a aproximação entre os setores da saúde e educação, por meio de estratégias indutoras tais como o Projeto Uma Nova Iniciativa (Projeto UNI - 1990), orientadas para a formação multiprofissional, o fortalecimento de componentes curriculares que desenvolvessem ações na comunidade e a valorização dos serviços de saúde nos processos formativos. Outra destas estratégias foi o Programa Nacional de Incentivo às Mudanças Curriculares para as Escolas Médicas (PROMED), que propunha a adequação da formação médica ao SUS a partir de reformas curriculares e de estágios em serviços públicos de saúde $e^{6,7,5}$.

A formulação de políticas de reorientação da formação para o SUS teve como sustentáculo a criação da Secretaria de Gestão do Trabalho e da Educação na Saúde (SGTES) no ano de 2003, que estreitou as relações entre as instituições formadoras e o sistema público de saúde e criou novos projetos indutores de mudanças, como o Vivências e Estágios na Realidade do Sistema Único de Saúde - VER-SUS 5 .

A partir do ano de 2005, a articulação entre os Ministérios da Saúde (MS) e da Educação (MEC) ganhou consistência, com a estruturação da parceria de cooperação técnica entre esses ministérios para a formação e desenvolvimento de recursos humanos na área da saúde. Instituiu-se também o Programa Nacional de Reorientação da Formação Profissional em Saúde (Pró-Saúde), visando implementar inovações na formação e ampliar as práticas educacionais no SUS ${ }^{8,9,5}$.

No Brasil, o processo de reforma nos currículos de graduação, iniciado em 1996, assumiu a necessidade do desenvolvimento de vínculos consistentes entre a formação e o exercício profissional e tomou como elementos basilares a relação com o mundo do trabalho e a flexibilização curricular, possibilitando às IES a construção de diferentes percursos de formação. Em 1997, a Secretaria de Educação Superior (SESu) propôs a substituição do sistema de currículos mínimos pelo modelo pautado em competências, considerado capaz de se adaptar às dinâmicas condições de perfil profissional exigidas pela sociedade. A graduação passa ter o papel de formação inicial no processo contínuo de educação permanente, inerente ao mundo do trabalho. As proposições de mudanças foram amparadas pela Lei de Diretrizes e Bases da Educação, que consubstanciou a necessidade de um documento de referência - as Diretrizes Curriculares Nacionais para os Cursos de Graduação (DCNs) ${ }^{10-12}$.

A formalização dessas diretrizes foi coordenada pelo MEC, que convocou as instituições de ensino superior a enviarem propostas para sua elaboração e ampliou a discussão, por meio de consultas e audiências públicas. $\mathrm{Na}$ área da saúde, os movimentos de busca de mudanças na formação profissional possibilitaram a mobilização de diversos atores, com a articulação de discussões e elaboração de propostas para a formação a partir do novo modelo de atenção, expresso nas DCNs dos cursos de graduação. Esse modelo adotou como aspectos centrais: o foco das ações de cuidado na saúde e não na doença; a coexistência de ações individuais e coletivas de atenção; o trabalho em equipes multiprofissionais; a coexistência de conteúdos biológicos clássicos e de conteúdos sobre fatores sociais, culturais, comportamentais, psicológicos, ecológicos, éticos e legais; e a educação permanente ${ }^{12}$.

As diretrizes assumiram as proposições da Declaração Mundial sobre Educação Superior no Século XXI, que definem que os currículos sejam pautados no desenvolvimento de competências e baseados em abordagens contemporâneas de formação, compatíveis com referências nacionais e internacionais. O ensino superior em saúde passou a se comprometer com a formação de profissionais capazes de atuar com qualidade, eficiência e resolutividade no sistema público de saúde brasileiro, tendo como princípios orientadores a integralidade da atenção à saúde e o trabalho em equipe e em redes ${ }^{13-15}$.

Ao se pautar pelo desenvolvimento de competências e pela visão compreensiva de currículo, que considera as dimensões do aprendiz, dos grupos e dos sistemas educacionais, a educação superior orienta suas práticas pela criticidade e pela capacidade de articulação com os desafios e questões sociais, com o objetivo de promover relações democráticas a partir de uma perspectiva cidadã ${ }^{13,16}$.

As competências profissionais envolvem a integração de conhecimentos, habilidades e atitudes ${ }^{(1)}$ para a realização de ações características de uma profissão; são construídas no

(1) Os termos conhecimentos, habilidades e atitudes são adotados como equivalentes, respectivamente, às capacidades e atributos cognitivos, psicomotores e afetivos, propostos por Lima $^{17}$. 
diálogo entre a formação e o mundo do trabalho e respondem a padrões orientadores da prática profissional ${ }^{17}$.

Os conhecimentos são definidos pelos aspectos cognitivos vinculados às informações, aos procedimentos e estratégias, e aos marcos teóricos de referência. As habilidades são definidas como recursos e destrezas cognitivos, emocionais e psicomotores necessários à realização das atividades. As atitudes são disposições adquiridas que predispõem os indivíduos para a ação e são frequentemente associadas aos valores, que são referenciais éticos que guiam atuações do indivíduo diante de si mesmo e dos outros ${ }^{18}$.

O foco deste artigo é a formação graduada em Terapia Ocupacional, no que se refere à temática das competências profissionais, identificadas nas Diretrizes Curriculares Nacionais do Curso de Graduação em Terapia Ocupacional. As DCNs definem e orientam os princípios comuns aos cursos da área da saúde para a organização, desenvolvimento e avaliação dos projetos pedagógicos e singularizam as competências e habilidades ${ }^{(2)}$ específicas para a Terapia Ocupacional. Essas competências gerais eram comuns aos cursos de Biomedicina, Educação Física, Enfermagem, Farmácia, Fisioterapia, Fonoaudiologia, Medicina, Medicina Veterinária, Nutrição, Odontologia, Psicologia e Terapia Ocupacional, que tiveram suas diretrizes instituídas entre 2001 e 2004. Educação Física e Psicologia apresentavam pequenas diferenças nessas competências em relação aos demais cursos, que as apresentavam de forma idêntica entre si. Psicologia, Medicina e Farmácia instituíram novas diretrizes curriculares em 2011, 2014 e 2017, respectivamente, não se observando a adoção de novas competências gerais comuns. Os demais cursos permanecem compartilhando essas competências gerais ${ }^{19,20,21}$.

Após mais de 15 anos de implementação das DCNs da área da saúde, e embora não esteja definida uma política de reformulação, as avaliações de suas proposições evidenciam lacunas na articulação entre formação, necessidades de saúde e demandas do SUS e apontam a necessidade de sua análise e revisão. Em 2014, a publicação das novas DCNs para os cursos de Medicina, foi um fator impulsionador para que as demais profissões apresentassem propostas para o aprimoramento de suas diretrizes ${ }^{22,23}$.

Na Terapia Ocupacional, o movimento de reformulação tem como marco o Seminário "Diretrizes Curriculares Nacionais da Terapia Ocupacional", que desencadeou a realização de discussões loco-regionais, coordenadas pelos cursos de graduação e com a participação de todos os atores envolvidos com a formação profissional (associações, conselho, docentes, discentes, usuários e profissionais dos serviços). Os resultados dos encontros regionais foram apresentados em outubro de 2018, no XVI Encontro Nacional de Docentes de Terapia Ocupacional (ENDTO). Houve ainda a designação de uma comissão composta por docentes das cinco regiões brasileiras para a continuidade da elaboração da proposição das novas diretrizes. No ano de 2019, o documento resultante desse processo foi apresentado aos cursos de graduação para consulta. As contribuições foram reunidas e incorporadas ao documento. A versão final foi encaminhada a especialistas da área em Terapia Ocupacional com vistas à elaboração de uma proposta a ser encaminhada ao Conselho Nacional de Saúde e MEC.

$\mathrm{O}$ presente estudo se dedica à apresentação e discussão das competências profissionais preconizadas pelas DCNs do Curso de Graduação em Terapia Ocupacional, no que se refere à especificidade e identidade profissional, entendidas como aquilo que caracteriza a profissão e a singulariza em relação às demais. É fundamental o estudo e produção de análises sobre as diretrizes, que norteiam a formulação de currículos e parâmetros educacionais em Terapia Ocupacional desde o ano de 2001.

\section{OBJETIVO}

Este trabalho tem como objetivo central identificar e compreender a especificidade e identidade profissionais presentes nas competências apresentadas como específicas para terapeutas ocupacionais pelas DCNs do Curso de Graduação em Terapia Ocupacional.

\section{MÉTODO}

Realizaram-se a leitura aprofundada e a análise das DCNs, com foco nas competências propostas como específicas para terapeutas ocupacionais. Essas competências foram agrupadas e sistematizadas em categorias temáticas, de acordo com a especificidade profissional identificada, a partir das quais se elaboraram sínteses descritivas que contribuíram para a identificação de suas principais características e fragilidades e para a reflexão sobre a especificidade profissional presente no documento ${ }^{24}$.

\section{RESULTADOS}

Primeiramente, será apresentada a análise global do documento, com considerações sobre o perfil do egresso,

(2) Não se observa, nesses documentos, a definição dos termos "competências" e "habilidades" e tem-se uma imprecisão conceitual, visto que para a maioria dos autores que tratam da temática, as habilidades compõem as competências, juntamente com os conhecimentos e as atitudes. 
formação generalista, organização curricular, utilização de metodologias ativas e adoção da pedagogia das competências. Em seguida, serão apresentadas sínteses das competências gerais para as profissões da área da saúde e, por fim, as sínteses das competências específicas propostas para terapeutas ocupacionais pelas diretrizes.

\section{Caracterização das DCNs para a Terapia Ocupacional}

O perfil do egresso proposto nas DCNs visa a formação generalista, humanista, crítica e reflexiva. A organização curricular deve se dar a partir da integração de conhecimentos e da utilização de metodologias ativas de ensino e aprendizagem, e o desenvolvimento de atividades práticas específicas da Terapia Ocupacional (TO) deve ocorrer desde o início do curso, com complexidade crescente da observação à prática assistida ${ }^{20}$.

A formação generalista diz respeito à capacidade de exercer a profissão em todas as suas dimensões, no que se refere à atuação preventiva e clínica-terapêutica e ao conhecimento dos fundamentos históricos, filosóficos e metodológicos da TO e dos diferentes modelos de intervenção ${ }^{20}$. Esses aspectos se aproximam da definição de Delari Júnior ${ }^{25}$ do profissional generalista como aquele que, ao dominar os fundamentos de sua área, adquire aptidão para transitar por funções e campos de atuação distintos.

A organização curricular, pautada pela integração de conhecimentos, permite assegurar a flexibilidade, a diversidade e a qualidade da formação profissional, de forma a preparar o estudante para os desafios colocados pelas transformações da sociedade, do mercado de trabalho e das condições de exercício profissional. Diante desses desafios, discute-se também que o tradicional modelo de desenho curricular baseado no estabelecimento de disciplinas estanques, o modelo da "grade", é insuficiente. Tal modelo se coloca apenas como instrumento de transmissão de conhecimentos e informações e estrutura-se a partir de conjuntos de disciplinas organizadas em um ciclo básico e um ciclo profissionalizante, com a realização de estágios nos períodos finais do curso, separação entre teoria e prática e crença de que a primeira é base para a segunda ${ }^{26}$.

Para superar tais impasses, educadores vêm propondo a adoção do modelo da "matriz curricular", considerado preferencial pelas diretrizes nacionais para a área da saúde, pois nele os conteúdos são organizados em torno de eixos definidos a partir do perfil profissional pretendido e agrupados em módulos, áreas ou atividades, integrados entre si de forma constante, sistemática, mediada pelo docente e em crescente complexidade, com a criação de redes de saberes cognitivos, procedimentais e atitudinais ${ }^{14,26}$.
O modelo de matriz e a adoção de metodologias ativas devem resultar da construção coletiva dos projetos pedagógicos, orientados para a articulação entre ensino, pesquisa e extensão e a formação integral do estudante. Neste desenho, o estudante é considerado a figura central e ativa dos processos de ensino e aprendizagem e tem no professor um facilitador e mediador desses processos ${ }^{20}$.

Tomando as proposições contidas nas diretrizes e os documentos ${ }^{2,3,10,11,13,27,14}$ que as fundamentam, verificase sua filiação à pedagogia das competências, pela identificação de características como: a caracterização sobre o desenvolvimento de competências abrange mais que a aquisição de conteúdos e pressupõe habilidades para a vida e atitudes baseadas nos direitos humanos; o protagonismo do estudante no desenvolvimento dessas competências ocorre mediante sua exposição a situações complexas que permitam a articulação teórico-prática e a mobilização integrada de diferentes recursos para a proposição de intervenções diante das demandas da realidade vivenciada ${ }^{28,16}$.

\section{Competências gerais para as profissões da saúde nas DCNs}

As diretrizes propostas, no início dos anos 2000, definiram competências gerais, comuns a todos os cursos da área da saúde, referidas às seguintes temáticas ${ }^{19,20}$ :

1. Atenção à saúde: desenvolvimento de ações individuais e coletivas em todos os níveis de atenção, de forma integrada e contínua com as instâncias e serviços de saúde; capacidade de pensamento crítico e de análise e proposição de soluções de/para os problemas de saúde da população;

2. Tomada de decisões: uso apropriado do trabalho e dos equipamentos, procedimentos e práticas, com sistematização e decisão das condutas a partir de evidências científicas;

3. Comunicação: confidencialidade das informações relacionadas à equipe e aos usuários dos serviços; postura acessível; capacidade de se comunicar não verbal e verbalmente e através da escrita; capacidade de usar tecnologias de comunicação e informação;

4. Liderança: desenvolvimento da liderança no trabalho em equipe multiprofissional, com compromisso, responsabilidade, empatia, habilidade para tomar decisões e para comunicação e gerenciamento dos serviços;

5. Administração e gerenciamento: gerenciamento do trabalho, dos recursos físicos e materiais e da informação; 
6. Educação permanente: capacidade de aprender continuamente; compromisso com a própria educação e com a de futuros profissionais, com estímulo à mobilidade acadêmico-profissional.

\section{Competências específicas para terapeutas ocupacionais nas DCNs}

A análise do conjunto das competências específicas resultou em 3 subgrupos e levou em conta o grau de especificidade de cada competência, de um patamar mais geral e pouco específico para as definições mais próprias e identitárias da Terapia Ocupacional.

a) Competências genéricas: Este grupo reuniu os tópicos que não se remetem exclusivamente à profissão e podem ser desenvolvidos por terapeutas ocupacionais e outros profissionais da área da saúde ${ }^{(3)}$. Eles orientam que os profissionais devem:

1. Em relação ao contexto social: conhecer os fatores sociais, econômicos, culturais e políticos da vida do país, fundamentais à cidadania e à prática profissional; reconhecer a saúde como direito e atuar de forma a garantir a integralidade da assistência; compreender as relações saúde-sociedade, de exclusão-inclusão social e estigmatização; participar da formulação e implementação de políticas sociais; reconhecer as modificações nas relações societárias, de trabalho e comunicação em âmbito mundial e os desafios e impactos por elas trazidos;

2. Em relação à inserção e atuação profissional: inserirse nos diversos níveis de atenção à saúde; conhecer a atuação int0er, multi e transdisciplinar e transcultural e conduzir processos terapêuticos interdisciplinares; desenvolver atividades de assistência, ensino, pesquisa, planejamento, gestão, assessoria e consultoria; conhecer métodos e técnicas de investigação e elaboração de trabalhos acadêmicos e científicos; conhecer a estrutura anátomo-fisiológica, cinesiológica e psíquica do ser humano e seu desenvolvimento; e desenvolver habilidades pessoais e atitudes necessárias para a prática profissional;

b) Competências com especificidade profissional intermediária: As proposições expressas neste grupo $^{(4)}$ abordam conhecimentos e habilidades presentes no exercício da Terapia Ocupacional que, embora se remetam diretamente à profissão, não descrevem os aspectos que qualificam o núcleo de sua identidade e singularidade profissional. Dentre eles, destacam-se: a emancipação e autonomia da população atendida como principais objetivos a serem atingidos pelos planos de ação e tratamento; a utilização do raciocínio terapêutico ocupacional; o conhecimento integrado do processo saúde-doença e de sua influência para a vida de relação e produção; o conhecimento e análise da estrutura social brasileira em relação ao perfil de produção e de ocupação dos indivíduos; o conhecimento das políticas sociais e da inserção do terapeuta ocupacional nesse processo; o conhecimento das realidades regionais e das prioridades assistenciais para formulação de estratégias de intervenção em Terapia Ocupacional; o conhecimento da problemática das populações que apresentam dificuldades temporárias ou permanentes de inserção e participação na vida social; o conhecimento dos princípios éticos que norteiam a prática e a pesquisa em Terapia Ocupacional; o conhecimento dos principais métodos de avaliação e registro, formulação de objetivos, estratégias de intervenção e verificação da eficácia das ações propostas em Terapia Ocupacional; o conhecimento das principais modalidades de atendimentos em Terapia Ocupacional; a atuação como agente social de transformação junto às comunidades, baseada nas noções de complementaridade e inclusão; o conhecimento das bases conceituais das terapias pelo movimento e da tecnologia assistiva e acessibilidade; e o desenvolvimento de atividades profissionais com as populações alvo, em diferentes equipamentos sociais e de saúde;

c) Competências específicas da Terapia Ocupacional: As proposições expressas neste grupo ${ }^{(5)}$ relacionamse à identidade e singularidade da Terapia Ocupacional e as diferenciam das demais profissões, ao abordar conhecimentos e habilidades referentes aos objetivos e objetos específicos da profissão: fundamentos históricos, filosóficos e metodológicos da Terapia Ocupacional e seus diferentes modelos de intervenção; compreensão do processo de construção

(3) Tópicos II, III, IV, V, VI, VII, XI, XVII, XIX, XXI, XXIV, XXXI, XXXII, XXXIII e XXXIV do Art. $5^{\circ}$ das Diretrizes Curriculares Nacionais do Curso de Graduação em Terapia Ocupacional (Brasil, 2002).

(4) Tópicos I, X, XII, XIII, XIV, XV, XVI, XX, XXII, XXIII, XXV, XXVII, XXVIII, XIX e XXX do Art. $5^{\circ}$ das Diretrizes Curriculares Nacionais do Curso de Graduação em Terapia Ocupacional (Brasil, 2002).

(5) Tópicos VIII, IX, XVIII e XXVI do Art. $5^{\circ}$ das Diretrizes Curriculares Nacionais do Curso de Graduação em Terapia Ocupacional (Brasil, 2002). 
do fazer humano e de como o homem se reconhece e reconhece sua ação; identificação, análise e interpretação das desordens da dimensão ocupacional do ser humano e utilização das diferentes atividades humanas como instrumentos de intervenção.

Essa categorização evidenciou que, das 34 competências apresentadas como específicas, 15 trazem características e princípios orientadores gerais, que podem se referir a outras profissões da saúde, e outras 15 expressam conhecimentos e habilidades referentes ao exercício da profissão sem, no entanto, apresentar aspectos identitários da Terapia Ocupacional em sua descrição. Apenas 4 dessas competências singularizam a profissão, diferenciando-a das demais.

\section{DISCUSSÃO}

As proposições das DCNs para os cursos de Terapia Ocupacional e sua adoção representaram, por um lado, avanço e alinhamento da área com o conjunto das profissões de saúde e com a construção do SUS, além de terem fomentado o desenvolvimento de reformas curriculares e a criação de currículos inovadores. Além disto, já se identificava clara tendência à adoção do paradigma psicossocial, em especial no que se refere ao respeito aos direitos de cidadania, à importância da atuação contextualizada, à atuação como agentes de transformação social e à prática centrada nas pessoas atendidas pela profissão.

Por outro lado, as diretrizes apresentam fragilidades, sendo a principal a ausência de singularidade na definição das competências propostas como específicas, com implicações para a afirmação da identidade da profissão em relação às demais. $\mathrm{O}$ estudo e análise do documento permitiram identificar outras problemáticas:

4. Apresentação desarticulada de diferentes temas e proposições na descrição das competências, com ausência de áreas de competência e/ou áreas temáticas centrais que pudessem agrupá-las e organizá-las;

5. Indefinição conceitual e indiferenciação entre os conceitos de competências e seus componentes conhecimentos, habilidades e atitudes - que devem ser desenvolvidos pelo currículo e compor o perfil do egresso;

6. As competências específicas presentes nas DCNs não estabelecem parâmetros que possam identificar, credenciar ou avaliar o perfil dos terapeutas ocupacionais esperado para os egressos dos cursos de graduação.
Na nova construção identitária da profissão, mais claramente alinhada com o paradigma psicossocial, a Terapia Ocupacional coloca como seu objetivo central a promoção de processos de inclusão social, a partir da compreensão dos contextos e condições de vida das pessoas e coletividades, do engajamento político e da defesa dos direitos humanos. Trata-se de uma inclusão social baseada na participação das pessoas atendidas e no seu engajamento em ocupações desejadas e aspiradas, e na criação de condições efetivas para a redução de barreiras e para promoção de acesso e participação social de todos ${ }^{29,30}$.

Essa mudança paradigmática não está claramente presente nas DCNs, embora estas sinalizem a importância da compreensão dos contextos globais e locais, da saúde como direito, dos movimentos de exclusão e inclusão social e, de forma restrita, dos processos de construção do fazer humano. No entanto, fazem-no de forma incipiente, desarticulada e, principalmente, inespecífica, estabelecendo poucas relações das competências com os objetos e objetivos da Terapia Ocupacional, o que se deve, como já exposto, à ausência de especificidade nas competências apresentadas como específicas. A quase totalidade dessas competências não se articula com a compreensão das ocupações, de como os contextos e dinâmicas sociais interferem no engajamento em ocupações e na participação social das pessoas atendidas pela profissão, da dimensão coletiva desse engajamento e do seu potencial de transformação social.

Além disto, a inexistência de parâmetros que atribuam singularidade e especificidade ao perfil esperado para terapeutas ocupacionais recém-formados e permitam, a partir disto, o credenciamento ou avaliação desses profissionais, dificulta a construção de processos de acreditação da profissão. A acreditação é um processo de garantia de qualidade por meio do qual se credenciam cursos que atendam a determinados padrões educacionais estabelecidos pelas autoridades responsáveis, e tem como principais contribuições: manter padrões nacionais consistentes; garantir a qualidade da educação profissional; apoiar o crescimento e desenvolvimento de cursos de terapia ocupacional e da profissão; monitorar os padrões educacionais internacionais para identificar tendências; e garantir possibilidades de mobilidade na graduação ${ }^{31}$.

\section{CONCLUSÃO}

As DCNs representaram importante avanço em relação aos parâmetros educacionais anteriores, evidenciado, entre outros aspectos, pelo alinhamento às demais profissões da saúde, pela formação voltada ao sistema público de saúde brasileiro e pela valorização de conhecimentos que 
possibilitam a compreensão da sociedade. Elas embasaram, também, o desenvolvimento de reformas curriculares e a criação de currículos inovadores, já se observando a adoção do paradigma psicossocial nas proposições do documento.

Por outro lado, essas diretrizes apresentam imprecisão conceitual sobre competências e seus elementos constitutivos e inespecificidade na definição das competências que trazem como específicas da profissão. Colocam-se desafios para o campo da formação em Terapia Ocupacional brasileiro, com destaque para as novas proposições das competências profissionais para terapeutas ocupacionais, dentro do processo de reformulação das Diretrizes Curriculares Nacionais, de forma que elas consigam contemplar práticas assistenciais e de ensino já presentes no contexto brasileiro e conferir especificidade às competências a serem desenvolvidas na formação dos terapeutas ocupacionais, que contemplem essa mudança paradigmática e a identidade profissional construída a partir dela. Alinha-se a isso a necessidade de que as novas proposições dialoguem com os parâmetros atualmente colocados pela Word Federation of Occupational Therapy para o credenciamento dos currículos nacionais e internacionalização do ensino e exercício profissional da área ${ }^{32}$.

No entanto, tais desafios não se encerram com a proposição de novas diretrizes, dada a conjuntura política de desmonte de políticas sociais e direitos humanos, em que se fundamentam as proposições e práticas da profissão, e considerando que a garantia da qualidade da formação e do desenvolvimento profissional exige a formulação e execução de proposições que articulem inovações curriculares e sistemas de avaliação e acreditação da profissão, o que ainda não ocorre para os cursos de Terapia Ocupacional no Brasil.

Contribuições das autoras: Marília Meyer Bregalda realizou a concepção e redação do texto e a análise do documento. Elisabete Ferreira Mângia realizou a concepção e revisão do texto e contribuiu com a análise do documento e a redação do texto.

\section{REFERÊNCIAS}

1. Brasil. Ministério da Saúde. Relatório Final da $8^{\mathrm{a}}$ Conferência Nacional de Saúde. Brasília; 1986. Disponívelem: http://bvsms. saude.gov.br/bvs/publicacoes/8_conferencia_nacional_saude_ relatorio_final.pdf.

2. Brasil. Senado Federal. Constituição da República Federativa do Brasil. Brasília; 1988. Disponível em: file://C:/Users/ ACER/Downloads/constituicao_federal_35ed.pdf.

3. Brasil. Ministério da Saúde. Lei $n^{\circ}$. 8.080, de 19 de setembro de 1990. Dispõe sobre as condições para a promoção, proteção e recuperação da saúde, a organização e o funcionamento dos serviços correspondentes e dá outras providências. Diário Oficial da União, Brasília; 1990. Disponível em: http:// conselho.saude.gov.br/legislacao/lei8080_190990.htm.

4. Ceccim RB, Armani TB, Rocha CF. O que dizem a legislação e o controle social em saúde sobre a formação de recursos humanos e o papel dos gestores públicos, no Brasil. Ciência e Saúde Coletiva. 2002;7(2):373-83. doi: 10.1590/S141381232002000200016 .

5. Dias HS, Lima LD, Teixeira M. A trajetória da política nacional de reorientação da formação profissional em saúde no SUS. Cien Saúde Coletiva. 2013;18(6):1613-1624. doi: 10.1590/ S1413-81232013000600013.

6. Brasil. Ministério da Saúde. Portaria interministerial $n^{\circ} 610$, de 26 de março de 2002. Institui o Programa Nacional de
Incentivo às Mudanças Curriculares para as Escolas Médicas. Diário Oficial da União, Brasília; 2002. Disponível em: https://www.jusbrasil.com.br/diarios/496301/pg-75-secao-1diario-oficial-da-uniao-dou-de-01-04-2002.

7. Zanotti, SJS. A participação popular na ampliação da esfera pública: o caso do Projeto UNI - uma nova iniciativa na formação dos profissionais de saúde [Dissertação]. São Paulo: Fundação Getúlio Vargas - FGV; 2003.

8. Brasil. Ministério da Saúde. Ministério da Educação. Portaria $\mathrm{n}^{\circ} 2.118$, de 03 de novembro de 2005. Institui parceria entre o Ministério da Educação e o Ministério da Saúde para cooperação técnica na formação e desenvolvimento de recursos humanos na área da saúde. Diário Oficial da União, Brasília; 2005. Disponível em: http://download.inep.gov.br/download/superior/2005/ avaliacao_institicional/portaria_interministerial_2118.pdf.

9. Brasil. Ministério da Saúde. Portaria interministerial $\mathrm{n}^{\circ} 2.101$, de 3 de novembro de 2005. Institui o Programa Nacional de Reorientação da Formação Profissional em Saúde - Pró-Saúde para os cursos de graduação em Medicina, Enfermagem e Odontologia. Diário Oficial da União, Brasília; 2005. Disponível em: https://www.portaleducacao.com.br/conteudo/artigos/ odontologia/portaria-interministerial-ms-mec-n-2101-de-3de-novembro-de-2005/3454.

10. Brasil. Lei no 9.394/96, de 20 de dezembro de 1996. Estabelece as diretrizes e bases da educação nacional. Diário Oficial da 
União, Brasília; 1996. Disponível em: http://www.planalto. gov.br/ccivil_03/leis/19394.htm.

11. Brasil. Ministério da Educação. Conselho Nacional de Educação/ Câmara de Educação Superior. Parecer n ${ }^{\circ} 776$, de 3 de dezembro de 1997. Orientação para as diretrizes curriculares dos cursos de graduação. Brasília; 1997. Disponível em: http://portal.mec.gov.br/setec/arquivos/pdf_legislacao/superior/ legisla_superior_parecer77697.pdf.

12. Moreira COF, Dias MSA. Diretrizes Curriculares na saúde e as mudanças nos modelos de saúde e de educação. ABCS Health Sciences. 2015;40(3):300-305. doi: 10.7322/abcshs.v40i3.811.

13. Organização das Nações Unidas para a Educação, a Ciência e a Cultura-UNESCO. Educação: um tesouro a descobrir. Relatório para a UNESCO da Comissão Internacional sobre a educação para o século XXI. Brasília; 1998. Disponível em: http://dhnet.org.br/ dados/relatorios/a_pdf/r_unesco_educ_tesouro_descobrir.pdf.

14. Brasil. Ministério da Educação. Conselho Nacional de Educação/ Câmara de Educação Superior. Parecer n ${ }^{\circ} 583$, de 4 de abril de 2001. Orientação para as Diretrizes Curriculares dos Cursos de Graduação. Brasília; 2001. Disponível em: http://portal.mec.gov.br/cne/arquivos/pdf/CES0583.pdf.

15. Oliveira GCC. Emergência de realidades no ensino superior da saúde: atos e vozes da área de fisioterapia nas Diretrizes Curriculares Nacionais. [Tese]. Porto Alegre: Universidade Federal do Rio Grande do Sul - UFRGS; 2011.

16. Organização das Nações Unidas para a Educação, a Ciência e a Cultura - UNESCO. International Bureau of Education. A conceptual framework for competencies assessment. Genebra/Suíça; 2016. Disponível em: file://C:/Users/ACER/ Downloads/245195eng.pdf.

17. Lima VV. Competência: distintas abordagens e implicações na formação de profissionais de saúde. Interface - Comunicação, Saúde, Educação. 2005;9(17): 369-379. doi: 10.1590/S141432832005000200012 .

18. Rué J. A formação por meio de competências: possibilidades, limites e recursos. In: Arantes, VA. Educação e competências. São Paulo: Summus; 2009. p. 15-74.

19. Brasil. Ministério da Educação. Parecer n ${ }^{\circ} 1.210$ do CNE/CES, de 10 de dezembro de 2001. Diretrizes Curriculares Nacionais dos Cursos de Graduação em Fisioterapia, Fonoaudiologia e Terapia Ocupacional. Diário Oficial da União, Brasília; 2001. Disponível em:http://portal.mec.gov.br/cne/arquivos/ pdf/pces1210_01.pdf.

20. Brasil. Ministério da Educação. Resolução no 3 do CNE/CES, de 19 de fevereiro de 2002. Institui Diretrizes Curriculares Nacionais do Curso de Graduação em Terapia Ocupacional. Diário Oficial da União, Brasília; 2002. Disponível em: http://portal.mec.gov.br/cne/arquivos/pdf/CES062002.pdf.

Recebido em: 01.12.2020

Aceito em: 19.03.2021
21. Brasil. Ministério da Educação. Diretrizes Curriculares - Cursos de Graduação. s/d. Disponível em: http://portal.mec.gov.br/ component/content/article?id=12991.

22. Brasil. Ministério da Educação. Resolução $n^{\circ} 6$ do CNE/CES, de 20 de junho de 2014. Institui Diretrizes Curriculares Nacionais do Curso de Graduação em Medicina e dá outras providências. Diário Oficial da União, Brasília; 2014. Disponível em: http:// www.fmb.unesp.br/Home/Graduacao/resolucao-den-2014.pdf.

23. Costa DAS, Silva RF, Lima VV, Ribeiro ECO. Diretrizes curriculares nacionais das profissões da Saúde 2001-2004: análise à luz das teorias de desenvolvimento curricular. Interface Comunicação, Saúde, Educação. 2018;22(67): 1183-1195. doi:10.1590/1807-57622017.0376.

24. Gibbs G. Análise de dados qualitativos. Porto Alegre: Artmed; 2009.

25. Delari Júnior A. Cinco critérios para a formação do psicólogo: da coerência ética à competência técnica. In: VIII Jornada Internacional de Psicologia. Umuarama, 2004. Resumo expandido. Disponível em: https://www.yumpu.com/pt/ document/view/12858563/cinco-criterios-para-a-formacaodo-psicologo-vigotski-brasil.

26. Anastasiou LGC. Grade e matriz curricular: conversas em torno de um desafio presente na educação superior. In: Freitas ALC, et al. Capacitação docente: um movimento que se faz compromisso. Porto Alegre: Edipucrs; 2010. p. 181-200.

27. Brasil. Lei n ${ }^{\circ} 10.172$, de 9 de janeiro de 2001. Aprova o Plano Nacional de Educação e dá outras providências. Diário Oficial da União, Brasília; 2001. Disponível em: www.planalto.gov.br/ ccivil_03/leis/leis_2001/110172.htm.

28. Perrenoud P. Construir as competências desde a escola. Porto Alegre: Artes Médicas; 1999.

29. Mângia EF, Barros JO. Projetos Terapêuticos e serviços de saúde mental: caminhos para a construção de novas tecnologias de cuidado. Rev Ter Ocup Univ São Paulo. 2009;20(2):85-91.

30. Organização Mundial de Saúde - OMS. Centro Colaborador da Organização Mundial da Saúde para a Família de Classificações Internacionais. CIF: Classificação Internacional de Funcionalidade, Incapacidade e Saúde. São Paulo: EDUSP; 2003.

31. Canadian Association of Occupational Therapists. Accreditation. s/d.Disponível em: http://www.caot.ca/site/ accred/accreditation?nav=sidebar.

32. World Federation of Occupational Therapists - WFOT. Minimum Standards for the Education of Occupational Therapists, Revised, 2016. Disponível em: https://www.wfot. org/assets/resources/COPYRIGHTED-World-Federationof-Occupational-Therapists-Minimum-Standards-for-theEducation-of-Occupational-Therapists-2016a.pdf. 